This item was submitted to Loughborough's Research Repository by the author.

Items in Figshare are protected by copyright, with all rights reserved, unless otherwise indicated.

\title{
Neuro-cognitive mechanisms of global Gestalt perception in visual quantification
}

PLEASE CITE THE PUBLISHED VERSION

https://doi.org/10.1016/j.neuroimage.2018.07.026

PUBLISHER

Elsevier

VERSION

AM (Accepted Manuscript)

\section{PUBLISHER STATEMENT}

This paper was accepted for publication in the journal Dalton Transactions and the definitive published version is available at https://doi.org/10.1016/j.neuroimage.2018.07.026

\section{LICENCE}

CC BY-NC-ND 4.0

\section{REPOSITORY RECORD}

Bloechle, J, S Huber, E Klein, Julia Bahnmuller, Korbinian Moeller, and J Rennig. 2018. "Neuro-cognitive Mechanisms of Global Gestalt Perception in Visual Quantification”. Loughborough University. https://hdl.handle.net/2134/12625349.v1. 


\section{Neuro-cognitive mechanisms of Gestalt perception in visual quantification}

Johannes Bloechle ${ }^{1,2,3}$, Stefan Huber ${ }^{1}$, Elise Klein ${ }^{1}$, Julia Bahnmüller ${ }^{1}$, Korbinian Moeller ${ }^{1,2,4}$, Johannes Rennig ${ }^{1,3,5}$

${ }^{1}$ Leibniz-Institut für Wissensmedien, Neuro-cognitive Plasticity Lab, Tuebingen, Germany ${ }^{2}$ Department of Psychology, University of Tuebingen, Tuebingen, Germany ${ }^{3}$ Centre of Neurology, Division of Neuropsychology, Hertie-Institute for Clinical Brain Research, University of Tuebingen, Tuebingen, Germany

${ }^{4}$ LEAD Graduate School and Research Network, University of Tuebingen, Tuebingen, Germany ${ }^{5}$ Department of Neurosurgery, Baylor College of Medicine, Houston TX, USA

* These authors contributed equally to the study and should be considered shared first authors

Address for correspondence:

Johannes Bloechle

Knowledge Media Research Center Tuebingen

Neuro-cognitive Plasticity Lab

Schleichstr. 6

72076 Tuebingen

Germany

j.bloechle@iwm-tuebingen.de 


\begin{abstract}
Recent neuroimaging studies identified posterior regions in the temporal and parietal lobe as neuro-functional correlates of subitizing as well as of Gestalt perception. Beyond notable overlap on a neuronal level Gestalt perception and subitizing are remarkably similar on a behavioral level because both processes represent a specific form of visual top-down processing where single elements are rapidly integrated into a superordinate entity. In the present study, we investigated whether subitizing draws on principles of Gestalt perception enabling rapid top-down processes of visual quantification. To this end, we designed two functional neuroimaging experiments: i) a functional localizer identifying voxels responding to global Gestalt stimuli in posterior temporo-parietal brain regions and ii) a visual quantification task on dot patterns with magnitudes within and outside the subitizing range. We hypothesized that voxels activated in Gestalt perception should respond stronger to dot patterns within than those outside the subitizing range. Results indicated that left-hemispheric posterior temporo-parietal brain regions associated with Gestalt perception indeed showed significantly stronger signals for dot patterns within compared to those outside subitizing range. Additionally, we trained a classifier with result patterns from Gestalt perception to predict neural responses of visual quantification within and outside the subitizing range. With this multivariate analysis approach we were able to classify in left and right hemispheric TPJ ROIs whether the brain actually processed a trial requiring subitizing using a classifier trained on Gestalt perception. The present study thus demonstrates that mechanisms of subitizing seem to build on processes of high-level visual perception.
\end{abstract}

\title{
Keywords
}

Gestalt; subitizing; number processing; object perception; human; TPJ 


\section{Significance Statement}

Previous neuroimaging studies on Gestalt perception and subitizing indicated considerable overlap of neural activation. However, contributions of neural mechanisms of high-level vision such as Gestalt perception to top-down process of visual quantification (i.e., subitizing) have never been evaluated specifically. Therefore, we pursued this in the current study representing a unique combination of research on neuroscience of visual perception and number processing. Results indicated joint activation for Gestalt perception and subitizing in temporo-parietal brains areas reflecting contributions of Gestalt perception to subitizing. Thereby, our present results showed for the first time that neural mechanisms of high-level vision are involved in visual quantification. 


\section{Introduction}

Gestalt perception describes a holistic form of object processing where local parts are visually integrated into a global entity. Gestalt processing is responsible to gain a holistic percept of the visual world (Wertheimer, 1923) and is involved in visual processes, like scene perception (Dalrymple et al., 2013; Shakespeare et al., 2013) or perception of artificial stimuli, like Navon letters (Navon, 1977). It has been demonstrated that global perception precedes processing of local parts (Navon, 1977) and can be particularly impaired in patients suffering from simultanagnosia (Bálint, 1909; Wolpert, 1924). Studies with simultanagnosia patients (Balslev et al., 2014; Friedman-Hill et al., 1995; Himmelbach et al., 2009; Huberle and Karnath, 2006; Luria, 1959) as well as functional neuroimaging work of global processing (Himmelbach et al., 2009; Huberle and Karnath, 2012; Rennig et al., 2015, 2013; Weissman and Woldorff, 2005; Zaretskaya et al., 2013) identified posterior temporo-parietal brain regions as neural correlates of Gestalt perception.

On a behavioral level, Gestalt perception seems comparable to a top-down mechanism of visual quantification called 'subitizing' (Kaufman et al., 1949; Wender and Rothkegel, 2000). In this process, a visual magnitude, presented for instance as a pattern of dots, can be estimated holistically without serial counting individual local elements (Trick and Pylyshyn, 1994). This top-down mechanism is highly dependent on the presented quantity of elements and usually works up to a number of four elements (Wender and Rothkegel, 2000). Several behavioral studies already linked the mechanism of subitizing to automatic visual pattern recognition (Ashkenazi et al., 2013; Logan and Zbrodoff, 2003; Mandler and Shebo, 1982; Wolters et al., 1987) - a process highly similar to visual Gestalt perception. Mandler and Shebo (1982) claimed that the spatial arrangement of up to four elements creates recognizable visual patterns (e.g., two dots form a line; three dots a triangle, four dots a rectangle) that are processed by a specific pattern recognition system, thereby enabling fast and precise quantification.

Processes of visual quantification within and outside the subitizing range have also been distinguished on a neuro-functional level. While quantification outside the subitizing range was found to engage superior parietal (Demeyere et al., 2012; He et al., 2014; Vuokko et al., 2013) and frontal (Vuokko et al., 2013) brain regions, the neural correlates of subitizing were associated with posterior temporo-parietal (Demeyere et al., 2014; He et al., 2014; Vetter et al., 2011; Vuokko et al., 2013) and occipito-parietal areas (Demeyere et al., 2012). Interestingly, the neural correlates of subitizing overlap considerably with activation patterns found in neuroimaging studies investigating Gestalt perception (Himmelbach et al., 2009; 
Huberle and Karnath, 2012; Rennig et al., 2015, 2013). A particular brain region associated with Gestalt perception (Huberle and Karnath, 2012; Rennig et al., 2015, 2013) as well as subitizing (Ansari et al., 2007; He et al., 2014; Vetter et al., 2011; Vuokko et al., 2013) is the temporo-parietal junction (TPJ).

Based on a body of work indicating a strong connection between Gestalt perception and subitizing we hypothesized that visual mechanisms of Gestalt perception should support topdown processes of visual quantification. To examine this hypothesis we designed two independent fMRI (functional magnetic resonance imaging) experiments: a functional localizer to identify brain regions involved in Gestalt perception (Huberle and Karnath, 2012; Rennig et al., 2015, 2013) and our main experiment requiring visual quantification of dot patterns. In the Gestalt localizer, we used stimuli that required specific Gestalt processes of visual grouping where single elements had to be integrated into a superior geometrical form (Navon, 1977). The visual quantification experiment comprised enumeration of dot patterns within and outside the subitizing range. In particular, we hypothesized that voxels identified as being activated in Gestalt perception (by the Gestalt localizer task) should contribute stronger to visual quantification for dot patterns within as compared to those outside the subitizing range in the independent visual quantification experiment. A stronger involvement of areas associated with visual Gestalt perception in the subitizing compared to quantification outside the subitizing range would demonstrate a significant contribution of mechanisms of visual perception to top-down visual quantification. This, in conclusion, would provide an explanation for the behavioral and neuro-cognitive differences between subitizing and visual quantification outside the subitizing range identified in previous studies.

\section{Materials and Methods}

\section{Participants}

24 right-handed volunteers $(16$ women, mean age $=24$ years; $\mathrm{SD}=6)$ participated in the study. Written informed consent was obtained from all participants. The study was approved by the Ethics Committee of the Medical Faculty of the University of Tuebingen. All participants had normal or corrected to normal vision and reported no previous history of neurological or psychiatric disorders.

Stimuli and Procedure

Gestalt Localizer Task. We used Gestalt stimuli from a previous study by Huberle and Karnath (2012) investigating neural correlates of Gestalt perception. Gestalt stimuli were 
global circles and squares that were constructed from local images of circles or squares. Analog to previous studies (Huberle and Karnath, 2012; Rennig et al., 2015), both congruent (e.g., global circle constructed from local circles) and incongruent (e.g., global circle constructed from local squares) Gestalt stimuli were used. Stimuli were scrambled by randomly exchanging a certain percentage of local elements. As the global Gestalt of the stimuli was easily perceived in the $20 \%$ scrambling condition (97\% correct) and global Gestalt perception was notably disturbed in the $80 \%$ scrambling condition (52\% correct, Huberle \& Karnath, 2012) we used a set of stimuli with these scrambling levels (see Figure 1 A). Participants saw 64 stimuli of each condition. Intact and scrambled Gestalt stimuli were presented for $300 \mathrm{~ms}$ followed by a blank screen with a central fixation cross for 2,700 ms. During the blank period, participants were required to indicate by button press whether the local elements formed a global circle or a global square. Responses with one of two MRcompatible buttons in the left and right hand were balanced for the expected responses. Additionally, $20 \%$ blank periods were randomly presented over the experiment. The blank periods ranged from 750 to $1250 \mathrm{~ms}$. During these additional blank periods a central fixation cross was presented. All Gestalt stimuli had a horizontal and vertical extension of $10^{\circ}$ visual angle.

Visual Quantification Task. Dot pattern arrangements were adapted from Wender \& Rothkegel (2000). To evaluate whether brain areas associated with Gestalt perception are particularly responsive during quantification of dot patterns with salient figural arrangements, random and canonical dot patterns were used in the visual quantification task. For such canonical dot patterns, frequently perceived in the same configuration, faster reaction times and reduced error rates were reported (Krajcsi et al., 2013; Mandler and Shebo, 1982; Wender and Rothkegel, 2000). The numerosity of canonical and random dot patterns ranged from two to eight. For each numerosity, three canonical and three random patterns were generated. Each of the two runs comprised 168 quantification trials, resulting in 336 critical trials in total. Dot patterns were presented in black against white background. The diameter of the dots was independent from numerosity and identical over all stimuli (2.5 ${ }^{\circ}$ visual angle). All dot patterns used in the present study are depicted in Figure 1B.

Experimental trials started with a central fixation cross. Subsequently, the critical dot pattern was presented for $200 \mathrm{~ms}$ followed by $2000 \mathrm{~ms}$ of visual noise preventing afterimages. Participants had to respond by pressing one of two MRI compatible response buttons placed in their left and right hand, once they had recognized the numerosity of the presented dot 
pattern. After the noise mask a number pad appeared on the screen for $3000 \mathrm{~ms}$ and participants had to navigate to the respective number. With the right hand button they navigated to the right, with the left one they navigated to the left within the number pad. Each trial was followed by a jittered inter-trial-interval of one second on average (ranging from 750 $\mathrm{ms}$ to $1250 \mathrm{~ms}$ ). Due to the rapid stimulus presentation it was not possible to enumerate dot patterns outside the subitizing range by serial counting and participants had to rely on an approximation strategy in these trials. Therefore, the term 'estimation' will be used throughout the course of the present report for these trials.

Stimuli of both experiments were projected on a screen above the head of the participant. Participants viewed the stimuli through a mirror mounted on the head coil of the scanner.

\section{[FIGURE 1 ABOUT HERE]}

\section{Eye Tracking}

To ensure that eye movement patterns did not differ between the two stimulus conditions in the Gestalt localizer (20 and $80 \%$ scrambled) as well as the subitizing and estimation ranges we recorded eye movements during all fMRI sessions with an MR compatible tracking device (EyeLink 1000 Plus, SR Research Ltd., Ottawa, Ontario, Canada). Prior to each run of functional imaging the eye tracker was calibrated. Preprocessing of eye tracking data included selection of stimulus presentation periods and saccade/fixation detection. Afterwards, the distance of gaze from the fixation cross was calculated for every sample. For the Gestalt localizer, we sorted the distance values per subject for trials with 20 and $80 \%$ scrambling. For the visual quantification experiment, we first sorted the data for each subject by dot quantity (subitizing and estimation). Only gaze data recorded during the entire time of stimulus presentation were considered for analyses later on; fixation periods, cue events and response periods were discarded from the analysis. Five subjects had to be excluded from the eye tracking analysis due to poor data quality.

\section{MRI Acquisition}

MRI data were acquired using a 3T Siemens Magnetom Prisma MRI system (Siemens AG, Erlangen, Germany) equipped with a 64-channel head coil. A high resolution T1-weighted anatomical scan $\left(\mathrm{TR}=2300 \mathrm{~ms}\right.$, matrix $=256 \times 256 \mathrm{~mm}^{2}, 176$ slices, voxel size $=1.0 \times 1.0 \times$ $1.0 \mathrm{~mm}^{3} ; \mathrm{FOV}=256 \mathrm{~mm}, \mathrm{TE}=2.92 \mathrm{~ms}$; flip angle $=8^{\circ}$ ) was acquired at the end of the experimental sessions. The functional measurements covered the entire brain using standard 
echo-planar-imaging (EPI) sequences (EPI; TR = $2400 \mathrm{~ms}$; TE $=30 \mathrm{~ms}$; flip angle $=80^{\circ}$; FOV $=220 \mathrm{~mm}, 88 \times 88$ matrix; 42 slices, voxel size $=2.5 \times 2.5 \times 3.0 \mathrm{~mm}^{3}$, slice gap $=10$ \%). Participants underwent the Gestalt localizer and the visual quantification task in two separate experiments and separate functional runs to prevent spurious effects due to dependent noise between data sets (Kriegeskorte et al., 2009). Total scanning time was approximately 60 minutes.

\section{Functional MRI Data Analysis}

fMRI data analyses were performed using SPM12 (http://www.fil.ion.ucl.ac.uk/spm). The first ten functional images of each participant were discarded to allow for magnetic saturation. For motion correction all remaining images of a participant were spatially realigned to his/her mean functional image. Realigned images were then normalized into standard stereotaxic MNI space (Montreal Neurological Institute, McGill University, Montreal, Canada) using the respective T1-weighted template. Images were resampled every $2.5 \mathrm{~mm}$ using 4th degree spline interpolation and smoothed with a $5 \mathrm{~mm}$ FWHM Gaussian kernel to account for residual intersubject variability in brain anatomy and to increase signal-to-noise ratio in the functional images. Data were high-pass filtered with a cut-off of $128 \mathrm{~s}$ to remove lowfrequency noise components and corrected for autocorrelation assuming an AR(1) process. Brain activity was convolved over all experimental trials with the canonical hemodynamic response function (HRF) and its first time derivative as implemented in SPM12.

For the functional Gestalt localizer, predictors for the two experimental conditions - intact and disturbed global Gestalt - were constructed by a convolution of stimulus onsets for $20 \%$ and $80 \%$ scrambled objects with the hemodynamic response function. The resulting design matrices comprised 2 experimental regressors, one for each scrambling level.

For the visual quantification experiment, we constructed predictors for each numerosity ( 2 to 8 ) in canonical and random arrangements, resulting in 14 experimental regressors.

Additionally, we included six motion parameters (roll, pitch, yaw; linear movement into x-, y, z-directions) as regressors of no interest in the functional localizer and the quantification experiment to capture residual movement-related artifacts. We used the individual participants' contrast images obtained from the first-level analysis for the second-level analysis. For the second level analysis we contrasted estimated beta weights for visual quantification trials within subitizing range against trials within estimation range using the flexible factorial design option within SPM12. All contrasts of the whole brain analysis were 
masked with the all $\mathrm{F}$ contrast. Voxels that passed a $p<.05$ (FWE) correction were reported as significant.

\section{fMRI functional localizer experiment}

The functional Gestalt localizer scan series was used to identify brain regions associated with global Gestalt perception in each participant; these regions of interest (ROIs) were then applied to the data collected in the visual quantification experiment. In order to restrict activated voxels to a pre-defined area already known to be involved in Gestalt processing we applied a functional mask to this analysis adapted from Huberle and Karnath (2012, see Figure 2). The individual Gestalt perception ROIs were created extracting active voxels within this functional mask for each participant by contrasting the intact Gestalt condition (20 $\%$ scrambled) against baseline. All voxels passing a threshold of $p<.05$ were then included in the individual Gestalt ROI (see Figure 2). Two out of 24 participants showed only left and three only right hemispheric activation within the functional mask. The average size of the individual Gestalt ROI was $1318 \mathrm{~mm}^{2}\left(\mathrm{SD}=717 \mathrm{~mm}^{2}\right)$ in the left hemisphere and $1856 \mathrm{~mm}^{2}$ $\left(\mathrm{SD}=1212 \mathrm{~mm}^{2}\right)$ in the right hemisphere. In case of bilateral ROIs we split these into a left and right hemispheric ROI for the later data analysis. The mean center of mass was located at the MNI coordinates $\mathrm{x}=-55(\mathrm{SD}=5) ; \mathrm{y}=-39(\mathrm{SD}=12) ; \mathrm{z}=24(\mathrm{SD}=4)$ for individual left hemispheric Gestalt ROIs and $\mathrm{x}=51(\mathrm{SD}=7) ; \mathrm{y}=-53(\mathrm{SD}=10) ; \mathrm{z}=28(\mathrm{SD}=5)$ for right hemispheric Gestalt ROIs. The reason for splitting up the ROIs by hemisphere was based on prior evidence about laterality of functions of Gestalt perception, visual quantification/subitizing as well as number processing. While processes of Gestalt perception were observed to be localized more strongly in the right hemisphere (Fink et al., 1996; Huberle and Karnath, 2012; Rennig et al., 2015; Zaretskaya et al., 2013), neural correlates of subitizing were identified to be more pronounced in both, areas of the left (Demeyere et al., 2014; Vuokko et al., 2013) as well as right hemisphere (He et al., 2014; Vetter et al., 2011; Vuokko et al., 2013).

\section{fMRI visual quantification experiment}

Each participant completed two functional runs of visual quantification in the scanner. Each run lasted 25 minutes. Within each run, dot pattern arrangements were presented for $200 \mathrm{~ms}$ in a rapid event-related fMRI design. Each combination of numerosity and arrangement was presented four times within each functional run resulting in 48 presentations for each numerosity. 
Mean percent signal changes (PSC) relative to baseline (fixation periods) within each participant's individual Gestalt perception ROI identified by the localizer task were extracted for canonical and random dot patterns within subitizing and estimation range using the MarsBar toolbox (http://marsbar.sourceforge.net).

\section{[FIGURE 2 ABOUT HERE]}

\section{Results}

Behavior

\section{Visual quantification.}

Behavioral results involved response times (RT) and error rates (ER). Analyses of RT were based on trials with correct responses only. Trials with RTs below 200ms were excluded. As the distribution of RTs was skewed to the right, we applied a log transformation (Ratcliff, 1993) resulting in a normal distribution of the data. Afterwards we additionally excluded log transformed RTs outside an interval of \pm 3 SD around a participant's mean. We used linear mixed effects models (LME) to analyze the log transformed RT data and generalized linear mixed effects models (GLME) with a binomial error distribution and the logit as link function to analyze ER data utilizing the R package lme4 (Bates et al., 2014). Fixed effects in these models were the categorical predictors dot pattern arrangement (i.e., canonical vs. random) and numerosity (i.e., 2, 3, 4, 5, 6, 7, and 8). All predictors were effect coded. We ran the LME with maximal random effects structure (Barr et al., 2013). In the GLME, we included a random intercept for participants. Due to technical problems with RT recordings, RT data of four participants could not be analyzed. Therefore, data of 20 participants entered RT analysis and data of 24 participants entered ER analysis.

RT Analysis. The LME revealed a significant interaction between dot pattern arrangement and numerosity $[F(6,22.20)=6.18, p<.001]$ depicted in Figure 3A. Post-hoc comparisons indicated that starting with four elements, $\log$ RT differed significantly between arrangements (i.e., 4 vs. 5,5 vs. 6,6 vs. 7, 7 vs. $8 ; z>2.81, p<.007$ ). Below four elements log RT did not differ significantly between arrangements (i.e., 2 vs.3, 3 vs. $4 ; z<0.93, p>.416$ ). Moreover, $\log$ RT of consecutive numerosities differed significantly for four against five elements and five against six elements in case of canonical arrangements $(z<-3.28, p<.004)$. However, in case of random arrangements, log RT differed additionally between three and four as well as between six and seven elements $(z<-2.50, p<.025)$. 
Moreover, we contrasted performance on canonical and random dot pattern arrangements in both number ranges: the estimation as well as the subitizing range (see Figure 3B). We observed significant differences in subitizing $(z=2.52, p=.012)$ as well as in estimation range $(z=7.37, p<.001)$.

\section{[FIGURE 3 ABOUT HERE]}

Furthermore, we observed significant main effects for dot pattern arrangement $[F(1,40.57)=$ $55.48 p<.001]$ and numerosity $[F(1,24.39)=11.11, p<.001]$. However, main effects should not be interpreted, because there was a significant difference between canonical and random arrangements only starting with four elements and $\log \mathrm{RT}$ differences between consecutive numerosities were not similar for canonical and random arrangement conditions.

ER Analysis. ERs showed a result pattern very similar to RTs (see Figure 3C). We observed a significant interaction between dot pattern arrangement and numerosity $\left[\chi^{2}(6)=94.94, p<\right.$ .001]. Post-hoc comparisons indicated that starting with five elements, error rates differed significantly between conditions $(z>4.60, p<.001)$. Comparisons within the subitizing range were not significant $(z<1.97, p>$.069). Moreover, error rates of consecutive numerosities differed significantly starting with four against five elements in case of random arrangements $(z<-4.92, p<.001)$. However, in case of canonical arrangements, error rates differed only between five and six elements $(z=-3.93, p<.001)$ and between seven and eight elements $(z=$ $-3.93, p=.014)$.

Moreover, we again contrasted performance on canonical and random arrangements in both number ranges: in estimation as well as in subitizing range (see Figure 3D). Specific contrasts indicated that canonical dot arrangements were significantly less error prone than random arrangements in the estimation range [estimated difference in $\log$ odds $=2.30$, in $\%: 17 \%, z=$ $11.27, p<.001$ ] but not in subitizing range [estimated difference in log odds $=-0.33$, in $\%:<$ $1 \%, z=-0.75, p=.469]$.

Both main effects of dot pattern arrangement $\left(\chi^{2}(1)=13.07, p<.001\right)$ and numerosity $\left(\chi^{2}(6)=\right.$ 444.05, $p<.001)$ were significant. Again, main effects should not be interpreted, because there was a significant difference between canonical and random arrangements only starting with five elements and error rates increased starting with the estimation range only in case of random arrangements. 


\section{Eye tracking}

For the Gestalt localizer, we calculated per trial the mean distance of gaze to the center of the screen as a measure of gaze position and the standard deviation of gaze position relative to center as a measure of eye movement distribution per subject for each of the two conditions (intact and disturbed global perception, i.e. 20 and $80 \%$ scrambled). For the visual quantification experiment, we compared eye movement behavior for subitizing and estimation ranges using the same variables. For the Gestalt localizer, we performed a $t$-test for both variables comparing average gaze position and gaze distribution between 20 and $80 \%$ scrambled stimuli. For both variables no significant differences were present (mean: $t(19)=$ $0.83, p=.42$; standard deviation: $t(19)=0.35, p=.73$ ). For the quantification experiment, we conducted the same analysis comparing average gaze position and gaze distribution for subitizing and estimation range. For both variables no significant differences were observable (mean: $t(19)=1.14, p=.28$; standard deviation: $t(19)=1.40, p=.20$ ).

\section{Neuroimaging}

ROI analysis

For the analysis of PSC values a linear mixed effect model (LME) was run with the categorical predictors dot pattern arrangement (canonical vs. random), numerosity (i.e., 2, 3, $4,5,6,7$, and 8 ) and hemisphere (left vs. right) as fixed effects and dot pattern arrangement and hemisphere as random effects. All predictors were effect coded. The $p$-values of the LME were calculated relying on the Kenward-Roger approximation for degrees of freedom available via the $\mathrm{R}$ package afex (Singmann et al., 2016). Again, $p$-values of subsequent posthoc tests were adjusted according to the Benjamini-Hochberg procedure (Benjamini and Hochberg, 1995) to account for multiple testing.

\section{[FIGURE 4 ABOUT HERE]}

The analysis of the PSC revealed a significant main effect of numerosity, $F(6,476)=3.78, p$ $<.001$ (see Figure 4A). Post-hoc comparisons indicated that PSC for numerosity "2" differed from PSC for other numerosities starting with four (i.e., 2 vs. 4, 2 vs. 5, 2 vs. 6, 2 vs. 7, 2 vs. $8 ; z>2.87, p<.019)$, whereas other post-hoc comparisons were not significant $(z<2.28, p>$ .082). Neither the main effect of dot pattern arrangement or hemisphere nor any other interaction was significant (all $p>.30$ ). Although we did not observe a significant interaction 
between the factors numerosity and hemisphere we want to use figure $4 \mathrm{C}$ to illustrate the data over all numerosities for both hemispheres separately.

\section{[FIGURE 5 ABOUT HERE]}

To evaluate how brain response in Gestalt ROIs differed between subitizing and estimation range, we employed a second LME with the categorical predictor number range (subitizing vs. estimation) replacing the predictor numerosity. For the categorical predictor subitizing we averaged PSC values for numerosities 2 to 4, the predictor estimation comprised numerosities 5 to 8 . This analysis revealed a significant interaction of number range and hemisphere, $F(1$, 488 ) $=4.24, p=.040$ (see Figure $4 \mathrm{D}$ ). The interaction indicated that the effect of number range was larger in the left hemisphere than in the right hemisphere (left $M=0.07, S E=0.02$, right $M=0.02, S E=0.02$ ). Post-hoc comparisons indicated that voxels in the left hemisphere involved in Gestalt perception contributed stronger to visual quantification in the subitizing than in the estimation range $(\mathrm{z}=3.88, p<.001)$ whereas no significant difference was observed for right hemispheric Gestalt ROIs $(z=1.02, p=.306)$. Moreover, the effect of hemisphere was significant for neither the subitizing $(z=1.56, p=.242)$ nor the estimation range ( $z=0.64, p=.523$ ) indicating a disordinal interaction. Therefore, the significant main effect of number range $(F(1,488)=12.16, p<.001)$ should not be interpreted (Figure $4 \mathrm{~B}$ illustrates this effect for a more comprehensive overview of our ROI results). Other main effects and interactions were not significant (all $p>.09$ ). This analysis indicated that voxels of the left hemisphere involved in Gestalt perception showed a stronger response during visual quantification in the subitizing than in the estimation range. The analysis with the predictor numerosity showed that this result pattern was neither driven by particularly strong activation in response to a specific numerosity in the subitizing range nor by particular weak activation in response to specific numerosities in the estimation range (see Figure $4 \mathrm{C}$ ).

\section{Multivoxel Pattern analysis (MVPA)}

Feature vectors from fMRI data were created by applying the approach suggested by Mumford and colleagues (2012). For every subject we calculated beta regression coefficient images for each item separately by running a general linear model including a regressor for the respective item as well as another regressor for all other items. Resulting beta values of voxels from individual Gestalt perception ROIs were then used as features when training support vector machines (SVM) to classify Gestalt trials correctly. Afterwards, we tested the 
performance of the trained SVMs in classifying subitizing and estimation trials (within the respective individual Gestalt ROIs) resulting in individual classification accuracy values per subject. We applied SVMs with a radial basis kernel. Moreover, we conducted grid search using 10 -fold cross validation to optimize the regularization parameter $\mathrm{C}=[0.001,0.01,0.1$, 1, 10, 100, 1000]. R package e1071 (Meyer et al., 2017) was used to train SVMs.

Performance of the SVMs was evaluated by running a GLME with dot pattern arrangement, hemisphere and their interaction as fixed effects, a random intercept for participants and accuracy as dependent variable. Again, we used the R package lme4 (Bates et al., 2014) for fitting GLME and we ran LRT for testing the significance of fixed effects relying on the R package afex (Singmann et al., 2015). We did not observe significant main effects for dot pattern arrangement $\left(\chi^{2}(1)=3.09, p=.079\right)$ and hemisphere $\left(\chi^{2}(1)=2.54, p=.111\right)$. Also the interaction between dot pattern arrangement and hemisphere was not significant $\left(\chi^{2}(1)=0.29\right.$, $p=.593)$. However, overall classification accuracy was significantly different from chance level $(\log$ odds $=0.13, S E=0.03$, in $\%: 53 \%, z=4.46, p<.001)$. This result indicated a significantly high similarity for neural representations of Gestalt perception and subitizing.

Whole brain analysis

Subitizing versus Estimation. Comparing subitizing with estimation revealed bilateral activation in angular gyrus (PGa), medial frontal cortex, and anterior cingulate cortex. Left hemispheric clusters were observed in middle temporal gyrus and cerebellum. Right hemispheric activation comprised clusters in inferior temporal gyrus, rolandic operculum (extending into the supramarginal gyrus) as well as precentral and postcentral gyrus (see Table 1 and Figure 5).

Estimation versus Subitizing. Contrasting brain activation for estimation and subitizing trials revealed bilateral activation of the IPS (hIP2, hIP3), insula and cerebellum. Left hemispheric activation was observed in inferior frontal gyrus, thalamus, postcentral sulcus, and basal ganglia (putamen, pallidum). Right hemispheric activation was found in middle frontal gyrus, middle cingulate cortex, as well as in inferior temporal gyrus and caudate nucleus (see Table1 and Figure 5).

Additionally, patterns of activated clusters were observed in motor-areas for both contrasts, reflecting manual responses after presentation of dot pattern arrangements in visual quantification trials. 


\section{[FIGURE 5 ABOUT HERE]}

\section{Discussion}

With the present study we pursued the question whether brain areas involved in Gestalt perception, which reflects a general ability of the human brain to perform top-down visual processes, also support subitizing, a specific form of top-down visual quantification. To investigate this question we conducted two functional neuroimaging experiments. In the Gestalt localizer we identified voxels in the temporo-parietal junction that were active during perception of visual stimuli requiring global Gestalt processing (Huberle and Karnath, 2012; Rennig et al., 2015, 2013). In the main visual quantification experiment behavioral and neural responses during visual quantification of dot patterns within the subitizing and estimation range were measured. Behavioral results indicated that participants were able to quantify dot patterns within the subitizing range significantly faster and with higher accuracy than dot patterns in the estimation range (Wender and Rothkegel, 2000). In a univariate analysis of our neuroimaging data we observed that those voxels associated with Gestalt perception in the localizer experiment were significantly more active in trials that allowed visual quantification within the subitizing range as compared to trials outside the subitizing range that had to be estimated. However, this significant difference was only present in left hemispheric TPJ ROIs. Moreover, a multivariate analysis of the neuroimaging data showed that a classifier trained on each individual's neuronal response pattern for Gestalt stimuli successfully classified result patterns from the independent visual quantification experiment as subitizing or estimation trials. We did not find differences in classification accuracy between left and right hemispheric ROIs. On a whole brain level, the contrast of subitizing versus estimation revealed activation mainly in bilateral posterior temporo-parietal brain areas including angular gyrus whereas the contrast of estimation versus subitizing showed bilateral activity in superior parietal extending into intraparietal sulcus as well as in frontal areas. Taken together, these results indicate a shared neural basis of Gestalt perception and quasi-simultaneous quantification within the subitizing range.

Our results associate neuronal mechanism of Gestalt perception with subitizing. This is in line with behavioral studies linking subitizing to visual pattern recognition, a process highly similar to Gestalt processing (Ashkenazi et al., 2013; Logan and Zbrodoff, 2003; Wolters et al., 1987). However, studies investigating a pattern recognition theory in subitizing mainly focused on the recognition of canonical (Ashkenazi et al., 2013) or particularly learned dot arrangements (Logan and Zbrodoff, 2003; Wolters et al., 1987). The present work extends the 
approach of linking subitizing to mechanisms of visual perception. Pattern recognition can be described as a more static form of top-down visual processing where perception of a given visual stimulus takes advantage of highly familiar patterns stored in memory (Logan, 1988; Logan and Zbrodoff, 2003). In contrast, the mechanisms behind Gestalt perception enable the human brain to perceive a variety of (previously unknown) complex visual stimuli, from Navon letters (Navon, 1977) to visual scenes (Dalrymple et al., 2013; Shakespeare et al., 2013). With the present results we were able to demonstrate that visual top-down mechanisms of subitizing are supported not only by memorizing familiar patterns but also mechanisms of visual Gestalt perception that are widely invariant to prior experience.

On a neural level the present findings are in good agreement with functional neuroimaging studies that associated Gestalt perception with posterior temporo-parietal brain areas in general (Himmelbach et al., 2009; Weissman and Woldorff, 2005; Yamaguchi et al., 2000) and TPJ in particular (Huberle and Karnath, 2012; Rennig et al., 2015, 2013). All these studies used different kinds of hierarchical stimuli where a global Gestalt had to be recognized from otherwise independent local elements. Thus, our finding that cortical areas associated with global Gestalt perception were activated more strongly during subitizing than estimation indicates a significant involvement of Gestalt perception in subitizing. As a consequence, it can be concluded that top-down visual quantification can also be considered a form of Gestalt perception by itself. Further empirical evidence for the link between the perceptual domain of Gestalt perception and subitizing comes from a recent study with patients suffering from developmental dyscalculia (Ashkenazi et al., 2013). In this study, it was dyscalculic patients who showed deficits in subitizing also showed significantly impaired implicit pattern recognition performance. This reflects, as mentioned above, a process highly similar to Gestalt perception. Similarly, it was found that the lower subitizing limit observed in children suffering from cerebral palsy - a neuro-motor developmental disorder - was associated with a general weakness of this patient group to perceive local elements of a visual array as a global Gestalt (Arp and Fagard, 2005).

Furthermore, our whole brain results are in line with previous studies investigating the neural correlates of processing non-symbolic magnitudes (i.e., dot patterns) within and outside the subitizing range. Subitizing was previously associated with posterior temporo-parietal (Demeyere et al., 2014; He et al., 2014; Vetter et al., 2011; Vuokko et al., 2013) and occipitoparietal areas (Demeyere et al., 2012). In particular, with our whole brain analysis we were able to replicate a recent fMRI study (Demeyere et al., 2014) that associated activation in the bilateral angular gyri, medial temporal cortex, areas in close vicinity of the precuneus, and 
posterior as well as anterior cingulate gyrus with subitizing using an fMRI adaptation paradigm. This remarkably precise replication using a different paradigm and different stimuli corroborates the contribution of the activated areas to subitizing.

Although several studies showed an association between TPJ activity and subitizing (Ansari et al., 2007; He et al., 2014; Vetter et al., 2011; Vuokko et al., 2013) this structure was not significantly involved in subitizing compared to estimation in our whole brain analysis. In our ROI analysis, however, we observed that regions in close vicinity of the TPJ (previously associated with Gestalt perception; e.g., Huberle and Karnath, 2012; Rennig et al., 2015, 2013) were involved significantly stronger in processing dot patterns within than those outside the subitizing range. Our findings identifying the IPS and (orbito-) frontal areas as correlates of estimation are in line with studies showing that perception of dot patterns outside the subitizing range engages superior parietal (Demeyere et al., 2012; He et al., 2014; Vuokko et al., 2013) and frontal areas (Vuokko et al., 2013).

Nevertheless, it is important to note that a study investigating functions of subitizing and enumeration in patients suffering from simultanagnosia observed a reverse result pattern than the present study and the referred body of literature (Dehaene and Cohen, 1994). From their behavioral experiments the authors concluded that patients with simultanagnosia were able to recognize small dot arrangements (1 to 2 elements) while they showed remarkable deficits for higher numbers of dots ( 3 - 6 elements) indicating preserved mechanisms of subitizing in simultanagnosia. While this conclusion held for within-subject comparisons for every patient, it did not for a comparison of the simultanagnosia patients with a control group. Compared to the control group, all patients showed significantly higher error rates for dot patterns larger than two and massively increased response times for dot patterns of one and two, but also for three or more dots. These results suggest serial processing of dot patterns already for those of very small numerosity in patients suffering from simultanagnosia and massive deficits of visual search that emerges for a larger amount of targets (see also Moeller et al., 2009 for similar results in children with dyscalculia). The data of Dehaene and Cohen (1994) therefore suggest that subitizing deficits in simultanagnosia do exist, which supports the findings of the present work.

Our univariate analysis showed that TPJ ROIs in the left hemisphere, which are involved in Gestalt perception showed significantly higher activation for subitizing compared to estimation. In contrast, the MVPA results indicated that TPJ areas associated with Gestalt perception from both hemispheres contributed significantly to subitizing. These results are consistent with studies showing absence and presence of hemispheric lateralization for Gestalt 
perception and subitizing. Processes of Gestalt perception were predominantly associated with the right hemispheric (Fink et al., 1996; Huberle and Karnath, 2012; Rennig et al., 2015; Zaretskaya et al., 2013) while neural correlates of subitizing were identified in areas of the left (Demeyere et al., 2014; Vuokko et al., 2013) and right hemisphere (He et al., 2014; Vetter et al., 2011; Vuokko et al., 2013). From this body of work and the results from our univariate analysis we can conclude that Gestalt mechanisms of the left hemisphere that have also been identified in several neuroimaging studies (Fink et al., 1997; Himmelbach et al., 2009; Huberle and Karnath, 2012) seem to support non-symbolic number processing. In contrast, the more sensitive multivariate analysis indicated significant involvement in subitizing in TPJ ROIs associated with Gestalt perception in both hemispheres. Due to a lower sensitivity of our univariate approach it may not be surprising that the univariate analysis did not identify mechanisms of Gestalt perception of the right hemisphere during subitizing. However, with the MVPA we were able to detect more fine-grained similarities between activation of voxels jointly activated during Gestalt perception and subitizing.

Due to different difficulty levels in conditions of subitizing and estimation a possible confound explaining the present results may be (visual) attention - especially when investigating posterior temporo-parietal brain areas (Corbetta and Shulman, 2002). It was observed that patients with lesions in the TPJ region show remarkable attention deficits such as spatial neglect (Hillis et al., 2005; Karnath et al., 2004, 2001) and extinction (de Haan et al., 2012). Moreover, fMRI studies revealed stronger activity in TPJ areas for tasks with high attentional demands like visual search (Himmelbach et al., 2006) or those requiring attential shifts in more demanding visual conditions (Doricchi et al., 2010; Thiel et al., 2004). However, these results indicate that differences in attentional processes may not explain the detected differences between subitizing and estimation in our TPJ ROIs consisting from voxels associated with Gestalt perception because subitizing is often seen as an effortless, automatic process with lower degree of difficulty engaging less attention (Piazza et al., 2003; Sathian et al., 1999; Trick and Pylyshyn, 1994 but see Pincham and Szucs, 2012; Railo et al., 2008). In contrast, estimation, which requires more attentional recources due to higher task complexity, elicited lower neuronal responses in our TPJ ROIs.

Behaviorally we observed a remarkable interaction between dot pattern arrangement and numerosity (see Figure 3A). However, this pattern was not reflected in the neural responses in the Gestalt ROIs. One might assume that particularly quantification of canonical arrangements recruits brain areas involved in Gestalt perception, because these arrangements reflect a more Gestalt-like spatial configuration. However, for dot patterns within the 
subitizing range the influence of the spatial arrangement might be negliable because dot patterns of up to four elements usually create recognizable visual patterns irrespective of the spatial arrangement (e.g., two dots form a line; three dots a triangle, four dots a rectangle; Krajcsi et al., 2013). Furthermore, the interactions in the behavioral data for ERs and RTs were driven by responses to stimuli outside the subitizing range with very similar behavioral responses for both kinds of arrangements within the subitizing range.

In the present study we demonstrated that areas in close vicinity to the TPJ that were significantly activated in Gestalt perception also showed stronger activation in subitizing. Thus, we suggest that subitizing takes advantage of processes underlying high-level visual perception. These results are in line with previous functional neuroimaging studies suggesting that areas involved in Gestalt perception (Himmelbach et al., 2009; Huberle and Karnath, 2012; Rennig et al., 2015, 2013) remarkably overlap with regions associated with subitizing, especially in the region of the TPJ (Ansari et al., 2007; He et al., 2014; Vetter et al., 2011; Vuokko et al., 2013), which was, however, previously not tested explicitly. Taken together, the present study shows that processes of higher-level visual perception are crucially involved in the quantification of small numerosities. 


\section{Acknowledgements}

This work was supported by the Leibniz-Competition Fund (SAW) providing funding for Elise Klein and supporting Stefan Huber, Johannes Bloechle and Julia Bahnmueller by a Margarete-von-Wrangell Fellowship appointed to Elise Klein (European Social Fund and the Ministry of Science, Research and the Arts Baden-Württemberg). The German Research Foundation (DFG) provided funding to Korbinian Moeller, Elise Klein and Johannes Rennig (MO 2525/2-1). Korbinian Moeller is principal investigator at the LEAD Graduate School [GSC1028], a project of the Excellence Initiative of the German federal and state governments.

\section{Conflict of interest}

The authors declare no competing financial interests. 


\section{References}

Ansari, D., Lyons, I.M., van Eimeren, L., Xu, F., 2007. Linking visual attention and number processing in the brain: the role of the temporo-parietal junction in small and large symbolic and nonsymbolic number comparison. J. Cogn. Neurosci. 19, 1845-53. doi:10.1162/jocn.2007.19.11.1845

Arp, S., Fagard, J., 2005. What impairs subitizing in cerebral palsied children? Dev. Psychobiol. 47, 89-102. doi:10.1002/dev.20069

Ashkenazi, S., Mark-Zigdon, N., Henik, A., 2013. Do subitizing deficits in developmental dyscalculia involve pattern recognition weakness? Dev. Sci. 16, 35-46. doi:10.1111/j.1467-7687.2012.01190.x

Bálint, R., 1909. Seelenlähmung des "Schauens", optische Ataxie, räumliche Störung der Aufmerksamkeit. Monatsschr. Psychiatr. Neurol. 25, 51-66.

Balslev, D., Odoj, B., Rennig, J., Karnath, H., 2014. Abnormal center-periphery gradient in spatial attention in simultanagnosia. J. Cogn. Neurosci. 26, 2778-88. doi:10.1162/jocn_a_00666

Barr, D.J., Levy, R., Scheepers, C., Tily, H.J., 2013. Random effects structure for confirmatory hypothesis testing: Keep it maximal. J. Mem. Lang. 68, 255-278. doi:10.1016/j.jml.2012.11.001

Bates, D., Maechler, M., Bolker, B., Walker, S., 2014. Fitting Linear Mixed-Effects Models using lme4. eprint arXiv:1406.5823 67, 51. doi:10.18637/jss.v067.i01

Benjamini, Y., Hochberg, Y., 1995. Controlling the false discovery rate: a practical and powerful approach to multiple testing. J. R. Stat. Soc. B 57, 289-300. doi: $10.2307 / 2346101$

Corbetta, M., Shulman, G.L., 2002. Control of goal-directed and stimulus-driven attention in the brain. Nat. Rev. Neurosci. 3, 201-15. doi:10.1038/nrn755

Dalrymple, K.A., Barton, J.J.S., Kingstone, A., 2013. A world unglued: simultanagnosia as a spatial restriction of attention. Front. Hum. Neurosci. 7, 145. doi:10.3389/fnhum.2013.00145

de Haan, B., Karnath, H.-O., Driver, J., 2012. Mechanisms and anatomy of unilateral extinction after brain injury. Neuropsychologia 50, 1045-53. doi:10.1016/j.neuropsychologia.2012.02.015

Dehaene, S., Cohen, L., 1994. Dissociable mechanisms of subitizing and counting: Neuropsychological evidence from simultanagnosic patients. J. Exp. Psychol. Hum. Percept. Perform. 20, 958-975. doi:10.1037/0096-1523.20.5.958 
Demeyere, N., Rotshtein, P., Humphreys, G.W., 2014. Common and dissociated mechanisms for estimating large and small dot arrays: value-specific fMRI adaptation. Hum. Brain Mapp. 35, 3988-4001. doi:10.1002/hbm.22453

Demeyere, N., Rotshtein, P., Humphreys, G.W., 2012. The neuroanatomy of visual enumeration: differentiating necessary neural correlates for subitizing versus counting in a neuropsychological voxel-based morphometry study. J. Cogn. Neurosci. 24, 948-64. doi:10.1162/jocn_a_00188

Doricchi, F., MacCi, E., Silvetti, M., MacAluso, E., 2010. Neural correlates of the spatial and expectancy components of endogenous and stimulus-driven orienting of attention in the posner task. Cereb. Cortex 20, 1574-1585. doi:10.1093/cercor/bhp215

Fink, G.R., Halligan, P.W., Marshall, J.C., Frith, C.D., Frackowiak, R.S., Dolan, R.J., 1996. Where in the brain does visual attention select the forest and the trees? Nature 382, 6268. doi:10.1038/382626a0

Fink, G.R., Marshall, J.C., Halligan, P.W., Frith, C.D., Frackowiak, R.S., Dolan, R.J., 1997. Hemispheric specialization for global and local processing: the effect of stimulus category. Proc. Biol. Sci. 264, 487-94. doi:10.1098/rspb.1997.0070

Friedman-Hill, S.R., Robertson, L.C., Treisman, A., 1995. Parietal contributions to visual feature binding: evidence from a patient with bilateral lesions. Science 269, 853-5. doi:10.1126/science.7638604

He, L., Zuo, Z., Chen, L., Humphreys, G., 2014. Effects of number magnitude and notation at 7T: Separating the neural response to small and large, symbolic and nonsymbolic number. Cereb. Cortex 24, 2199-2209. doi:10.1093/cercor/bht074

Hillis, A.E., Newhart, M., Heidler, J., Barker, P.B., Herskovits, E.H., Degaonkar, M., 2005. Anatomy of spatial attention: insights from perfusion imaging and hemispatial neglect in acute stroke. J. Neurosci. 25, 3161-7. doi:10.1523/JNEUROSCI.4468-04.2005

Himmelbach, M., Erb, M., Karnath, H.-O., 2006. Exploring the visual world: the neural substrate of spatial orienting. Neuroimage 32, 1747-59. doi:10.1016/j.neuroimage.2006.04.221

Himmelbach, M., Erb, M., Klockgether, T., Moskau, S., Karnath, H.-O., 2009. fMRI of global visual perception in simultanagnosia. Neuropsychologia 47, 1173-7. doi:10.1016/j.neuropsychologia.2008.10.025

Huberle, E., Karnath, H.-O., 2012. The role of temporo-parietal junction (TPJ) in global Gestalt perception. Brain Struct. Funct. 217, 735-46. doi:10.1007/s00429-011-0369-y Huberle, E., Karnath, H.-O., 2006. Global shape recognition is modulated by the spatial 
distance of local elements--evidence from simultanagnosia. Neuropsychologia 44, $905-$ 11. doi:10.1016/j.neuropsychologia.2005.08.013

Karnath, H.-O., Fruhmann Berger, M., Küker, W., Rorden, C., 2004. The anatomy of spatial neglect based on voxelwise statistical analysis: a study of 140 patients. Cereb. Cortex 14, 1164-72. doi:10.1093/cercor/bhh076

Karnath, H.O., Ferber, S., Himmelbach, M., 2001. Spatial awareness is a function of the temporal not the posterior parietal lobe. Nature 411, 950-953.

Kaufman, E.L., Lord, M.W., Reese, T.W., Volkmann, J., 1949. The Discrimination of Visual Number. Am. J. Psychol. 62, 498. doi:10.2307/1418556

Krajcsi, A., Szabó, E., Mórocz, I.Á., 2013. Subitizing is sensitive to the arrangement of objects. Exp. Psychol. 60, 227-34. doi:10.1027/1618-3169/a000191

Kriegeskorte, N., Simmons, W.K., Bellgowan, P.S.F., Baker, C.I., 2009. Circular analysis in systems neuroscience: the dangers of double dipping. Nat. Neurosci. 12, 535-40. doi:10.1038/nn.2303

Logan, G.D., 1988. Toward an instance theory of automatization. Psychol. Rev. 95, 492-527. doi:10.1037/0033-295X.95.4.492

Logan, G.D., Zbrodoff, N.J., 2003. Subitizing and similarity: toward a pattern-matching theory of enumeration. Psychon. Bull. Rev. 10, 676-82. doi:10.3758/BF03196531

Luria, A., 1959. Disorders of "simultaneous perception" in a case of bilateral occipito-parietal brain injury. Brain 82, 437-49.

Mandler, G., Shebo, B.J., 1982. Subitizing: An Analysis of Its Component Processes. J. Exp. Psychol. Gen. 111, 1-22. doi:10.1037/0096-3445.111.1.1

Meyer, D., Dimitriadou, E., Hornik, K., Weingessel, A., Leisch, F., Chang, C.-C., Lin, C.-C., 2017. Package “e1071”. Misc Functions of the Department of Statistics, Probability Theory Group (Formerly: E1071), TUWien.

Moeller, K., Neuburger, S., Kaufmann, L., Landerl, K., Nuerk, H.-C., 2009. Basic number processing deficits in developmental dyscalculia: Evidence from eye tracking. Cogn. Dev. 24, 371-386.

Mumford, J.A., Turner, B.O., Ashby, F.G., Poldrack, R.A., 2012. Deconvolving BOLD activation in event-related designs for multivoxel pattern classification analyses. Neuroimage 59, 2636-2643. doi:10.1016/j.neuroimage.2011.08.076

Navon, D., 1977. Forest before trees: The precedence of global features in visual perception. Cogn. Psychol. 9, 353-383. doi:10.1016/0010-0285(77)90012-3

Piazza, M., Giacomini, E., Le Bihan, D., Dehaene, S., 2003. Single-trial classification of 
parallel pre-attentive and serial attentive processes using functional magnetic resonance imaging. Proc. Biol. Sci. 270, 1237-45. doi:10.1098/rspb.2003.2356

Pincham, H.L., Szucs, D., 2012. Intentional subitizing: Exploring the role of automaticity in enumeration. Cognition 124, 107-116. doi:10.1016/j.cognition.2012.05.010

Railo, H., Koivisto, M., Revonsuo, A., Hannula, M.M., 2008. The role of attention in subitizing. Cognition 107, 82-104. doi:10.1016/j.cognition.2007.08.004

Ratcliff, R., 1993. Methods for dealing with reaction time outliers. Psychol. Bull. 114, 510532. doi:10.1037/0033-2909.114.3.510

Rennig, J., Bilalić, M., Huberle, E., Karnath, H.-O., Himmelbach, M., 2013. The temporoparietal junction contributes to global gestalt perception-evidence from studies in chess experts. Front. Hum. Neurosci. 7, 513. doi:10.3389/fnhum.2013.00513

Rennig, J., Himmelbach, M., Huberle, E., Karnath, H.-O., 2015. Involvement of the TPJ area in processing of novel global forms. J. Cogn. Neurosci. 27, 1587-600. doi:10.1162/jocn_a_00809

Sathian, K., Sim, T.J., Peterson, S., Patel, G.A., Hoffm, J.M., Grafton, S.T., 1999. Enumeration and Attention. J. Cogn. Neurosci. 11, 36-51.

Shakespeare, T.J., Yong, K.X.X., Frost, C., Kim, L.G., Warrington, E.K., Crutch, S.J., 2013. Scene perception in posterior cortical atrophy: categorization, description and fixation patterns. Front. Hum. Neurosci. 7, 621. doi:10.3389/fnhum.2013.00621

Singmann, H., Bolker, B., Westfall, J., Aust, F., Fox, J., Lawrence, M.A., 2016. Package “ afex ". Analysis of Factorial Experiments.

Thiel, C.M., Zilles, K., Fink, G.R., 2004. Cerebral correlates of alerting, orienting and reorienting of visuospatial attention: An event-related fMRI study. Neuroimage 21, 318 328. doi:10.1016/j.neuroimage.2003.08.044

Trick, L.M., Pylyshyn, Z.W., 1994. Why Are Small and Large Numbers Enumerated Differently? A Limited-Capacity Preattentive Stage in Vision. Psychol. Rev. 101, 80102. doi:10.1037/0033-295X.101.1.80

Vetter, P., Butterworth, B., Bahrami, B., 2011. A candidate for the attentional bottleneck: setsize specific modulation of the right TPJ during attentive enumeration. J. Cogn. Neurosci. 23, 728-36. doi:10.1162/jocn.2010.21472

Vuokko, E., Niemivirta, M., Helenius, P., 2013. Cortical activation patterns during subitizing and counting. Brain Res. 1497, 40-52. doi:10.1016/j.brainres.2012.12.019

Weissman, D.H., Woldorff, M.G., 2005. Hemispheric asymmetries for different components of global/local attention occur in distinct temporo-parietal loci. Cereb. Cortex 15, 870-6. 
doi:10.1093/cercor/bhh187

Wender, K.F., Rothkegel, R., 2000. Subitizing and its subprocesses. Psychol. Res. 64, 81-92. doi:10.1007/s004260000021

Wertheimer, M., 1923. Untersuchungen zur Lehre von der Gestalt. II. Psychol. Forsch. 4, 301-350. doi:10.1007/BF00410640

Wolpert, I., 1924. Die Simultanagnosie - Störung der Gesamtauffassung. Zeitschrift für die Gesamte Neurol. und Psychiatr. 93, 397-415.

Wolters, G., Van Kempen, H., Wijlhuizen, G.J., 1987. Quantification of small numbers of dots: Subitizing or pattern recognition? Am. J. Psychol. 100, 225-237. doi:10.2307/1422405

Yamaguchi, S., Yamagata, S., Kobayashi, S., 2000. Cerebral asymmetry of the "top-down" allocation of attention to global and local features. J. Neurosci. 20, RC72.

Zaretskaya, N., Anstis, S., Bartels, A., 2013. Parietal cortex mediates conscious perception of illusory gestalt. J. Neurosci. 33, 523-31. doi:10.1523/JNEUROSCI.2905-12.2013 


\section{Figure captions:}

\section{Figure 1:}

Stimuli used in the different experiments. (A) Illustration of the global stimuli adapted from the study of Huberle and Karnath (2012). Depicted are a circle and a global square in the $20 \%$ scrambling condition (intact global perception) and an example for the $80 \%$ scrambling condition (disturbed global perception). (B) Dot patterns adapted from Wender \& Rothkegel (2000) ranging from 2 to 8 in canonical and random spatial arrangement.

\section{Figure 2:}

Individual Gestalt ROIs in three representative subjects. Gestalt ROIs (red) contain voxels responsive to intact global Gestalt stimuli stronger than to baseline within the functional TPJ mask (green) from Huberle \& Karnath (2012). ROIs are superimposed on a 3D rendered surface and axial slices of the single-subject T1 MNI152 template. The figure shows the vertical z-coordinate for each slice of standardized MNI space.

\section{Figure 3:}

(A) Visual quantification time (RT in ms) for canonical and random dot pattern arrangements as a function of numerosity. A steep increase of RTs in the random condition for numerosities larger 4 was observed, reflecting a transition from subitizing to estimation processes. (B) Visual quantification times (RT in ms) for canonical and random dot pattern arrangements in the subitizing and estimation range. (C) Mean percent error for canonical and random dot pattern arrangements as a function of numerosity. (D) Mean percent error for canonical and random dot pattern arrangements in subitizing and estimation range. Error bars indicate the standard error of the mean of parameters.

\section{Figure 4:}

(A) Mean percent signal change (relative to fixation) in bilateral Gestalt ROIs as a function of numerosity. (B) Mean percent signal change in bilateral Gestalt ROIs in subitizing and estimation range. (C) Mean percent signal change in left (red) and right (green) hemispheric Gestalt ROIs as a function of numerosity. (D) Mean percent signal change in left and right Gestalt ROIs as a function of number range. Left-hemispheric voxels involved in Gestalt perception contribute stronger to visual quantification for dot patterns within compared to those outside the subitizing range $(* * *$ p-value $<.001)$ whereas this was not observed for 
right hemispheric Gestalt ROIs. Error bars indicate the standard error of the mean of parameters.

\section{Figure 5:}

Results of the whole brain analysis. The contrast between subitizing and estimation (depicted in red) revealed activity in bilateral angular gyrus, medial temporal cortex (in close vicinity of the precuneus), posterior cingulate gyrus, and anterior cingulate cortex (ACC). The contrast between estimation and subitizing trials (depicted in blue) revealed stronger activation in bilateral IPS, insula, middle, and inferior frontal areas as well as middle cingulate cortex (MCC). Contrasts were masked with the all-F-contrast. Voxels that passed a threshold of $p<$ .05 (FWE) are depicted on a 3D rendered surface and on coronal slices. 